Article

\title{
Ang Paglubog nina Hippokrates at Galen sa Kanluran: Isang Intepretasyon sa Anyo ng Siyantipikong Rebolusyon sa Larangan ng Medisina
}

F.P.A. Demeterio

\begin{abstract}
This paper conceptualizes the interaction of three discursive paths: the history of science, scientific education, and the debate about the Filipinization of scientific education. The paper analyzes the form of scientific revolution in the field of medicine which is different from its counterparts in the fields of astronomy and physics; as such, the paper contributes a particular narrative of that provides proof that it is possible to tackle scientific issues using the Filipino language as medium. It assumes that the Filipino language should be used in the teaching of science and in scientific research. The scientific revolution in medicine is seen as the eventual downfall of Hippocrates and Galen and the rise of the theories of Paracelsus, Vesalius, Fracastoro, and Harvey.

Keywords: Hippocrates, Galen, Filipino language, scientific revolution in medicine
\end{abstract}

\section{Introduksyon}

A

ng papel na ito ay isinulat sa interseksyon ng tatlong mas malawak na diskursibong landas: ang kasaysayan ng siyensya, ang edukasyong pangsiyensa, at ang usapin tungkol sa pagsasa-Filipino sa edukasyong pangsiyensya dito sa ating bansa. Bunsod sa masalimuot na pinagmumulan ng papel na ito, hinangad nitong tugunan ang apat na layunin.

Nangunguna dito ang pagsaliksik sa isang aspekto ng siyantipikong rebolusyon na kadalasang naisantabi dahil ang nakararaming pag-aaral sa historikal na penomenang ito ay nakatuon sa aspekto ng astronomiya at pisika at sa mga mala-higanteng imahen nina Nicholas Copernicus, Galileo Galilei, at Isaac 
Newton. Marahil dulot ito sa katotohanang mas mahirap paksain ang siyantipikong rebolusyon sa larangan ng medisina dahil hindi ganoon kalinaw kung ano nga ba ang tradisyunal na medisina na pinataub ng umuusbong na modernong alternatibo, o ano ang lumang paradigma na dramatikong pinalitan ng isang bagong paradigma, kapag gagamitin natin ang tanyag nang lengguwahe ng historyador at pilosopo ng siyensyang si Thomas Kuhn. Maski ang anyo at mga katangian ng umuusbong na modernong alternibo at ng bagong paradigma ay hindi rin ganoon kalinaw. Presuposisyon ng papel na ito na naiiba ang istraktura ng siyantipikong rebolusyon sa larangan ng medisina kung ihahambing ito sa istraktura ng mas pamilyar na siyantipikong rebolusyon sa larangan ng astronomiya at pisika. Kaya, kung maaaring ikuwento ang siyantipikong rebolusyon sa larangan ng astronomiya at pisika gamit ang iilan lamang prinsipal na tauhan, higit na mas kumplikado dito ang kuwento ng siyantipikong rebolusyon sa larangan ng medisina. Dagdag pa dito, ang matematikal na pagdalumat sa kalikasan na sinasabing isa sa mga pangunahing katangian ng siyantipikong rebolusyon ay hindi gaano katingkad sa unang mga yugto ng bagong medisina. ${ }^{1}$ Kaya, ninais ng papel na ito na intindihin nang lubusan ang anyo at mga katangian ng siyantipikong rebolusyon sa naturang larangan.

Pumapangalawa sa mga layunin ng papel na ito ay ang paglahad sa isang makabayang kamalayan na ang wikang Filipino, na sadyang ginamit sa pagsulat ng papel na nito, ay handang-handa nang tumalakay sa iba't ibang paksa sa domain ng siyensya. Ang makabayang kamalayang ito ay taliwas sa mas popular na kaisipan na kapag edukasyong pansiyantipiko na ang pag-uusapan, mas akma pa ring gamitin ang wikang Ingles kaysa wikang Filipino. Sa kasamaang palad ang popular na kaisipang ito ay tila sinusuportahan ng mga pahayag ng batikang lingguwistang si Bonifacio Sibayan (1916-2005), nang ilang ulit niyang sinabi na ang wikang Filipino ay hindi pa intelektuwalisado; at pati na ng mga mahahalagang probisyon ng ating pang-edukasyong ordinansang bilingguwalismo at multilingguwalismo, na nag-uutos sa atin na gamitin ang wikang Ingles para sa mga asignaturang siyensya, teknolohiya at matematika. Presuposisyon ng papel na ito na ang paggamit ng wikang Filipino ay magpapadali sa pedagohiya ng edukasyong pangsiyensa sa ating bansa. Para malampasan nating lahat ang mala-kumunoy na debate kung intelektuwalisado na nga ba o hindi pa ang wikang Filipino, ginamit ng papel na ito ang mga patakaran ng pagsasalin ng Sentro ng Wikang Filipino ng Pamantasan ng Pilipinas para maisa-Filipino ang pagsusuri tungkol sa siyantipikong rebolusyon sa larangan ng medisina. Simple ang mga probisyon ng patakarang ito: maghanap ng Filipino/Tagalog na katumbas; kapag walang Filipino/Tagalog na katumbas, maghanap ng katumbas mula sa iba't ibang lokal na wika ng Filipinas; kapag walang mahanap na katumbas mula sa iba't ibang lokal

${ }^{1}$ John Henry, The Scientific Revolution and the Origins of Modern Science (New York: Palgrave, 2002), 14.

(c) 2013 F.P.A. Demeterio

http://www.kritike.org/journal/issue 12/demeterio june2013.pdf

ISSN 1908-7330 
na wika, gamitin ang katumbas sa wikang Espanyol; kapag hindi pamilyar sa atin ang katumbas sa wikang Espanyol, gamitin na lamang ang wikang katumbas sa wikang Ingles. ${ }^{2}$

Pumapangatlo sa mga layunin ng papel na ito ay ang pag-aanyaya sa mga Pilipinong babasa nito na dahil mas malinaw at mas maiintindihan natin ang siyensya, o kasaysayan nito, o edukasyon nito, kapag ang ginagamit nating wika sa pagtatalakay ay ang ating itinuturing na wikang pambansa, nararapat lamang na unti-unti na nating suportahan ang pagsusulat, pagbabasa, pagtuturo, at pananaliksik sa siyensya at teknolohiya gamit ang parehong wikang ito. Naaalala ko dito ang malaking hamon na hinaharap ni Dr. Severino Capitan, isang propesor ng beterinarya sa Pamantasan ng Pilipinas-Los Banos at isa sa mga tagapagsalita sa pambansang seminar ng SANGFIL noong taong 2008. Si Dr. Capitan ay nagsusumikap gumawa ng isang translation software para maisalin sa wikang Filipino ang mga pananaliksik ng kanilang kolehiyo para mapakinabangan ito $\mathrm{ng}$ mga magsasaka na hindi nakababasa ng Ingles. Hindi na sana kinakailangan pa nating magpapakahirap gumawa ng translation software kapag deretsahan nating isinasagawa at isinusulat ang ating mga pananaliksik sa wikang naiintindihan ng ating mga kababayan. Presuposisyon ng papel na ito ang isang malawakang paggamit ng wikang Filipino sa domain ng siyensya at teknolohiya ay hindi lamang magpapaunlad sa mga domain na ito, kung hindi pati na sa ating ekonomiya at lipunan. Ito ay dahil ang wikang naiintindihan ng mas marami nating kababayan ay siyang magbubukas ng mga pagkakataong mapag-uusapan at mapapakinabangan ang mga kaalamang natutuklasan natin mula sa iba't ibang larangan ng ating pananaliksik.

Higit sa lahat hangarin ng papel na ito na makaaambag ng isang materyal na may nilalaman at midyum na maaaring magpapayaman sa edukasyong pangsiyensya ng ating mga mag-aaral sa sekondarya at kolehiyo. Kapag naiintindihan nila nang lubusan ang kasaysayan ng siyensya, maaaring ilan sa kanila ay maaakit sa halaga at hamon ng siyensya at kaluanan ay magpapasyang itaya ang kani-kanilang kinabukasan para maging mga masigasig na tagapagpalaganap ng siyensya at teknolohiya.

Para matugunan ang mga hangaring ito, ang papel na ito ay may tatlong substansyal na seksyon. Batay sa historyograpikal na prinsipyong ang kasaysayan ay isang konstrak lamang, ang una at ikalawang substansyal na seksyon ay mga pagtangkang gawan ng naratibo ang tradisyunal na medisina batay sa dalawang katauhan nina Hippokrates at Galen na silang tumatayong pundasyon ng klasiko at medyibal na medisina. Batay naman sa parehong historyograpikal na prinsipyo, ang ikatlong substansyal na seksyon ay isa ring pagtangkang gawan ng naratibo ang mga pangunahing batikus sa tradisyunal na medisina na nagmumula kina

\footnotetext{
mga Sining, 1996).

(c) 2013 F.P.A. Demeterio

http://www.kritike.org/journal/issue 12/demeterio june2013.pdf ISSN 1908-7330

${ }^{2}$ Virgilio Almario, Patnubay sa Pagsasalin (Manila: Pambansang Komisyon sa Kultura at 
Paracelsus, Vesalius, Fracastoro at Harvey, na unti-unting nagpalubog sa medisina nina Hippokrates at Galen at naglikha ng pangangailangang bumuo ng mga bagong teorya sa medisina. Inaasahan ng papel na ito ang tatlong magkarugtung na naratibo ay silang magsisilbing balangkas para masuri ang kasaysayan ng siyantipikong rebolusyon sa larangan ng medisina at maikuwento ito sa mga mambabasang sumasaklaw hindi lamang sa mga edukador ng siyensya kung hindi pati na rin sa mga mag-aaral ng siyensya mula sa sekondarya at kolehiyo.

Ang konklusyon ng papel na ito ay tatalakay sa mga aspekto na nagsisilbing dahilan kung bakit tunay ngang matatawag na rebolusyunaryo ang mga nangyaring pagbabago sa larangan ng medisina. Tutukuyin ng konklusyon ang mga elemento ng tradisyunal na medisina na naisantabi at nanatili. Ipapaliwanag din nito kung paano naiiba ang proseso ng pagpalit ng paradigma sa larangan ng medisina sa kaparehong proseso sa larangan ng astronomiya at pisika, gamit uli ang lengguwahe at mga konsepto ni Kuhn.

\section{Ang Medisinang Hippokratiko at Galeniko ng Sinaunang Panahon}

Kapag ang tradisyunal, o pre-modernong medisina ng medyibal na Europa ang pinag-uusapan, dapat lamang na mabanggit ang mga pangalan ng ilang mga personalidad na nangunguna sa pagtaguyod nito, katulad ng Persiyanong pilosopo at manggagamot na si Ibn Sina, o Avicenna (circa 980-1037); ng Arabong pilosopo at manggagamot na si Al-Kindi (circa 801-873); ng Hudyong pilosopo at manggagamot na si Isaac Israeli (circa 832-circa 932); ng Italyanong surgeon na si Roger Frugardi (circa 1140-circa1195); ng Italyanong surgeon, prayle at obispo na si Teodoric Borgognoni (1205-1298); ng Italyanong manggagamot, anatomista at surgeon na si Mondino de Luzzi (circa 1270-1326); at ilan pang mga batikang manggagamot, anatomista at surgeon. Ngunit nakamamanghang isipin na ang mga personalidad na humugis sa medisina sa panahong ito ay mga indibidwal na hindi naman talaga napabibilang sa medyibal na Europa. Ang mga ito ay na walang iba kung hindi sina Hippocrates (circa 460circa $370 \mathrm{BC}$ ), isang Griyegong namuhay noong ika-lima hanggang ika-apat na siglo bago ipinanganak si Hesus Kristo, at si Galen (129-circa $199 \mathrm{AD}$ ), isang Griyego-Romanong namuhay naman noong ikalawang siglo matapos ipinanganak si Hesu Kristo. Ang mga akda nina Hippocrates at Galen ang nagsilbing mga pangunahing teksto ng medyibal na Europyanong medisina. ${ }^{3}$ Kaya sa halip na talakayin natin ang mga naging kontribusyon ng mga Arabo, Hudyo at Italyanong manggagamot, anatomista at surgeon, mas maiiintindihan natin ang anyo ng

${ }^{3}$ Vivian Nutton, "The Fortunes of Galen," in Hankinson, R.J. The Cambridge Companion to Galen (Cambridge: Cambridge University Press, 2008), 362-372.

(C) 2013 F.P.A. Demeterio

http://www.kritike.org/journal/issue 12/demeterio june2013.pdf ISSN 1908-7330 
medyibal na medisina kapag tututukan na lamang natin ang mga teorya at kaalamang inilahad nina Hippocrates at Galen.

Si Hippokrates ay ipinanganak sa Griyegong isla ng Kos, mula sa isang Asclepiad na angkan, o mga kasapi sa grupo ng mga ritwalistiko at relihiyosong manggagamot na naniniwalang pinaka-ninuno nila si Asculapius, ang Griyegong dios ng panggagamot. Bilang isang Asclepiad, nakuha niya ang kanyang pundasyunal na kaalaman sa medisina mula sa kanyang ama, at nilinang at pinalawak niya ito sa pamamagitan ng kanyang paglakbay sa mainland ng Gresya, Thessaly, Thrace, at sa Karagatan ng Marmara. Bukod sa medisina, nag-aral din si Hippokrates ng retorika sa ilalim ni Georgias ng Leontini (circa 485-circa 380 $\mathrm{BC}$ ), at ng pilosopiya mula sa atomista at pilosopong si Demokritos ng Abdera (circa 460-circa 370).

Kinalala siya ngayon bilang ama ng medisina dahil sa kanyang pagtiwalag sa panguhaning prinsipyo ng mga Asclepiad at ng maraming Griyego noon na ang mga sakit at karamdaman ng tao ay ipinararanas sa kanila ng mga dios at diosa at kaya ang lunas para sa mga ito ay makakamtan sa pamamagitan ng pagdadasal at pag-aalay. Sa mas madaling salita, inilipat ni Hippokrates ang diskurso ng medisina mula sa relihiyon at mistisismo patungo sa diskurso ng rasyunalismo at naturalismo. Hanggang sa kasalukuyang panahon, ginagamit pa rin ng maraming bansa at ng maraming pamantasang medikal ang Hippokratikong panata para sa panunumpa ng mga bagong manggagamot.

Kapag susundin natin ang mga istriktong pamantayan ng kasalukuyang historikal na pananaliksik, masasabi nating walang malinaw na mga dokumentong naipamana si Hippokrates sa kasaysayan ng medisina. Sa halip, ang mayroon lamang tayo ay isang koleksyon ng mga animnapung teksto na nakasulat sa wikang Ionian na tinatawag natin ngayong "Hippocratic Corpus." Sinabi ni W.H.S. Jones, sa kanyang introduksyon sa Loeb na edisyon ng nasabing corpus, na samu't sari ang anyo at orihinal na silbi ng mga tekstong ito: may mga "textbook para sa mga manggagamot," may mga "textbook para sa mga layko," may mga "ulat mula sa ilang pananaliksik o materyal para sa ilang pananaliksik," may mga "panayam para sa mga mag-aaral ng medisina," may mga "sanaysay ng mga pilosopong may interes sa medisina," at may mga "notebook at scrapbook."4 Batay sa mga paksa, estilo ng pagkakasulat, at lohikal na pagkakaugnay ng mga tekstong ito, malinaw na hindi sila nagmumula sa iisang may-akda. Ang pinakarasonableng paninindigan ng mga skolar ngayon ay maaaring ilan sa mga tekstong ito ay talagang sulat nga ni Hippokrates, ngunit ang nakararami ay ambag ng kanyang mga mag-aaral at tagasunod, at may iilan pang nagmumula sa ibang sistema ng Griyegong panggagamot.

${ }^{4}$ W.H.S. Jones, “General Introduction," in The Loeb's Classical Library's Hippocrates, Vol. 1 (London: William Heinemann Limited), xxii.

(C) 2013 F.P.A. Demeterio

http://www.kritike.org/journal/issue 12/demeterio june2013.pdf ISSN 1908-7330 
Dahil sa sitwasyong ito, hindi maaaring alamin ninoman kung ano talaga ang mga pangunahing prinsipyo ng medisina ni Hippokrates sa pamamagitan ng deretsahang pagsusuri sa mga nilalaman ng Hippocratic Corpus. Kinakailangan muna niyang malaman ang pangkalahatang diskurso ng medisinang ito mula sa mga sinaunang Griyego, Romano, Hudyo at Arabong historyador at komentarista, bago pa man siya mag-umpisang maghanap ng mga detalye nito mula sa nasabing corpus.

Sentro sa medisina ni Hippokrates ang doktrina tungkol sa apat na humour, o mga likido ng katawan, na hiniram niya mula kay Alcmaeon ng Croton (circa 535-circa $470 \mathrm{BC}$ ). Itinuro ng doktrinang ito na ang katawan ng tao ay may apat na uri humour: ang dugo, ang itim na apdo, ang dilaw na apdo, at ang plema. Kapag taglay ng apat na humour ang isang pantay-pantay na proporsyon, o sitawasyong "demokrasya" sa wika ni Alcmaeon, taglay din ng isang indibidwal ang isang mabuting kalusugan. ${ }^{5}$ Subalit kapag isa sa mga humour ang mangingibabaw, o sitwasyong "monarkiya" sa wika ni Alcmaeon, o hindi kaya wala sa natural na pagkakapantay-pantay ang proporsyon ng mga ito, taglay ng isang indibidwal ang partikular na sakit o karamdaman. ${ }^{6}$ Para kay Alcmaeon at Hippokrates, ang panggagamot ay isang proseso ng paggabay sa katawan ng tao para makamtan muli nito ang natural na pagkakapantay-pantay ng proporsyon ng kanyang apat na humour. Para maibalik ang ng katawan ang natural na balanse ng mga humour, itinuturo ni Hippokrates ang paggamit ng ilang herbal na lunas; pagmanipula sa temperatura ng katawan; pagkain ng ilang piling potahe katulad ng lugaw na barley, hydromel (pulot at tubig), oxymel (pulot at suka) at alak; paggamit ng labatiba; at ang pagsagawa ng venesection o pagbabawas ng dugo. ${ }^{7}$

Naniniwala si Hippokrates na ang katawan ng tao ay may likas na kayahang lumaban sa anumang sakit at karamdaman. Kaya ang tungkulin ng isang manggagamot ay ang pag-aalalay lamang sa natural na kapangyarihang ito. Para maiwasan ang mga sakit at karamdaman, para mapanatili ang natural na balanse ng apat na humour, at para mapalakas lalo ang likas na kapangyarihan ng katawan na lumaban kapag dumapo man ang sakit at karamdaman, mahalaga para kay Hippokrates na bantayan palagi ang kalusugan ng katawan sa pamamagitan ng tamang diyeta at sapat na ehersisyo. May mga historyador na naniniwala na ang positibong pagtutok ni Hippokrates sa kalusugan, sa halip na sa negatibong sakit at karamdaman, ay isa sa mga pangunahing dahilan kung bakit naging matatag at laganap ang sistema ng medisinang kanyang ipinundar. ${ }^{8}$

\footnotetext{
${ }^{5}$ Francesca Spina, "The Medicine of Ancient Greece," in Facolta di Medicina e Chirurgia dell' Università degli Studi di Cagliari, <http://pacs.unica.it/biblio/lesson1.htm>, Date Accessed: 27 July 2011.

${ }^{6} \mathrm{Ibid}$.

${ }^{7}$ Victor Robinson, The Story of Medicine (New York: The Home Library, 1943), 50.

${ }^{8}$ Spina, "The Medicine of Ancient Greece."
} 
Kapansin-pansing wala masyadong diskusyon si Hippokrates tungkol sa ugnayan ng kalusugan, o karamdaman, sa anatomiya o pisyolohiya ng katawan. Ito ay dahil hindi siya nagkaroon ng pagkakataong mapag-aralan nang masinsinan ang anatomiya at pisyolohiya ng tao bunsod sa Griyegong taboo na nagbabawal sa pagsasagawa ng dissection, o ang paghimay sa isang bangkay, at ng vivisection, o ang paghimay sa katawan ng isang buhay na tao.

Si Galen, o Aelius Galenus, o Cladius Galenus, naman ay ipinanganak sa Pergamum, ang kasalukuyang Bergama ng bansang Turkey, may limang siglo na matapos mamatay si Hippokrates. Nagmula siya sa isang mayamang Griyegong angkan, at nakapag-aral at nakapagsanay sa medisina sa mga lunsod ng Pergamum, Smyrna, Corinthia, Crete, Cilicia, Cyprus at higit sa lahat sa Alexandria. ${ }^{9}$ Mahalaga ang pagdalaw ni Galen sa Alexandria, hindi lamang dahil sa ang lunsod na ito ay nagsisilbing sentro ng kultura at kaalaman ng Hellenistikong mundo, kung hindi dahil minsan nang dinaig sa lunsod na ito ang Griyegong taboo laban sa dissection at vivisection ng Egyptian na tradisyon sa paghimay ng bangkay na batayan naman sa kanilang proseso ng paggawa ng mummy. Sa katunayan ipinagmamalaki ng Alexandria ang kanyang batikang mga anatomista tulad nina Herophilus ng Chalcedon (circa 330-260 BC) at Erasistratus ng Chios (circa 304circa $250 \mathrm{BC}$ ). Kahit pa man hindi na naabutan ni Galen ang lantarang pagsasagawa ng dissection at vivisection doon sa Alexandria, dahil sa lubusang paglanap ng naturang Griyegong taboo na sinigundahan naman ng Romanong kaugalian, napakinabangan pa rin niya nang husto ang anatomikal na tradisyon ng lunsod na ito.

Nang bumalik siya sa Pergamum, nanungkulan siya bilang doktor ng mga gladyador, kung kailan lalong niyang napayaman ang kanyang kaalaman sa anatomiya sa pamamagitan ng mabilasang pagmatyag sa panloob na pisika ng mga sugatang gladyador at bangkay. ${ }^{10}$ Pumunta si Galen sa Roma kung saan nanilbihan siya bilang manggagamot sa imperial na korte nina Marcus Aurelius (121-180 AD), Lucius Verus (130-169 AD), Commodus (161-192 AD), at Septimius Severus (145-211 AD).

Isang mapagmuni-muning tao si Galen at naniniwalang ang medisina at pilosopiya ay dapat magkakasama para maging matatag ang intelektuwal at moral na katayuan ng isang doktor. ${ }^{11}$ Para sa kanya ang isang mahusay na manggamot ay dapat isang indibidwal na may malalim na pang-akademikong kaalaman na hinasa sa isang mahaba at replektibong aktuwal na panggagamot. Itinuring niya ang kanyang sarili bilang isang mabuting tagasunod ni Hippokrates, at bilang isang

\footnotetext{
${ }^{9}$ D.E. Eichholz, "Galen and his Environment," in Greece and Rome 20:59 (June 1951), 60.

${ }^{10}$ Julius Rocca, "Anatomy," in The Cambridge Companion to Galen (Cambridge: Cambridge University Press, 2008), 245.

${ }^{11}$ Eichholz, "Galen and his Environment," 63.
} 
Hippokratikong manggagamot tinanggap niya ang doktrina ng apat na humour bilang teorya ng kalusugan, ng sakit at karamdaman, at ng panggagamot.

Ngunit dahil batid ni Galen ang anatomikal at pisyolohikal na tradisyon ng Alexandria, sinikap niyang mapayaman ang Hippokratikong medisina gamit ang anatomiya at pisyolohiya. Iginigiit niya na dapat magsasagawa ang mga doktor ng dissection hindi lamang para mapatalas nila ang kani-kanilang husay sa surgery, kung hindi para na rin mapalawak at mapalalim nila ang kani-kanilang pagkakaunawa sa pisikal na istraktura ng katawan at kung paano ito gumagana. Subalit dahil sa umiiral na Griyego at Romanong taboo laban sa paghimay sa katawan ng tao, para sa kanyang sariling pagsasanay sa dissection at vivisection, ginamit niya ang mga hayop, katulad ng aso, baboy, at unggoy (barbary ape). Ang kaalamang kanyang nakalap mula sa mga hayop na ito ay kanyang isinailalim sa ilang modipikasyon batay sa kanyang limitadong kaalaman sa anatomiya ng tao na kanya namang nakalap bilang doktor ng mga gladyador doon sa lunsod ng Pergamon.

Mula sa kanyang regular na dissection at vivisection, natuklasan niya ang kahalagahan ng mga lamang loob (internal organs). Ngunit, dahil sa katotohanang nagmumula pa rin sa mga hayop ang kanyang kaalaman, nagkaroon siya ng maraming pagkakamali. Inakala niya, halimbawa, na ang sinapupunan ng babae ay pareho sa sinapupunan ng aso, o na ang posisyon ng rinyon (kidney) ng tao ay katulad sa posisyon ng rinyon ng baboy, o na ang istraktura ng utak ng tao ay kapareho sa istraktura ng utak ng baka o ng kambing.

Gayunpaman, napaunlad ni Galen ang Hippokratikong medisina. Halimbawa, sa halip na ipaliwanag ang lahat nang sakit at karamdaman gamit ang doktrina ng apat na humour, sinabi ni Galen na bukod sa mga sakit at karamdamang epekto ng kawalan ng balanse ng mga humour, may mga sakit at karamdaman ding epekto ng ilang diperensya sa mga internal at eksternal na organo ng katawan, at meron ding mga sakit at karamdamang epekto ng pagbagsak ng kaubuoang coherence ng katawan. ${ }^{12}$ Pinatalas din ni Galen ang Hippokratikong proseso ng diagnosis sa pamamagitan ng sistema sa pagdama sa pulso ng pasyente, pagsuri sa ihi, at pagmatyag sa pisikal na anyo ng mukha at kilos ng pasyente. ${ }^{13}$ Binigyang diin niya ang halaga ng case study at beside teaching ni Hippokrates na nagbigay ng karagdagang lalim at personal na interaksyon sa proseso ng panggagamot. Pinayaman niya ang mga herbal na lunas, ang prosesyo ng pagmanipula sa temperatura ng katawan, ang pagpili ng angkop na pagkain, at ang pagsagawa ng venesection na dati nang itinuturo ni Hippokrates. Higit sa lahat, isinasabuhay ni Galen ang kanyang paniniwala na dapat ang isang manggagamot ay isa ring surgeon.

${ }^{12}$ Philip Van der Eijk, “Therapeutics," in The Cambridge Companion to Galen, 295.

${ }^{13}$ Ibid., 294.

(c) 2013 F.P.A. Demeterio

http://www.kritike.org/journal/issue 12/demeterio june2013.pdf

ISSN 1908-7330

\section{(cc) BY-NC}


Isa sa mga pinakamahalagang teoretikal na sistemang itinuturo ni Galen ay ang kanyang teorya tungkol sa daloy ng dugo. Sinunod ni Galen ang masalimuot na eksplinasyon ng Griyegong pilosopo na si Aristotle (384-322 BC), na hinubog naman ng konsepto ng tripartite na kaluluwa ng kanyang guro na si Platon (429-347 BC). ${ }^{14}$ Dahil hindi pa na nadalumat noon ang konsepto ng sirkulasyon ng dugo, inisip ni Galen na may tatlong uri ng dugo na dumadaloy sa katawan ng tao: ang dugong nagmumula sa atay na dumadaloy sa mga vein na siyang responsable sa pagdadala ng sustansya sa iba't ibang parte ng katawan at sa pagpapalaki ng indibidwal; ang dugong nagmumula sa puso na dumadaloy sa mga artery na siyang responsable sa pagbibigay ng buhay sa katawan; at ang dugong nagmumula sa utak na dumadadaloy naman sa mga nerve na siyang responsable sa sensasyon, paggalaw at pag-iisip.

Nakamamangha ang kaalamang nakalap ni Galen tungkol sa nervous system, galaw at sensasyon, na nakuha niya sa pamamagitan ng vivisection at eksperimentasyon gamit ang mga hayop. ${ }^{15}$ Ngunit marami ang kanyang pagkakamali sa kanyang diskusyon tungkol sa dugo na dumadaloy sa artery at sa vein. Una, inakala niya na ang dugo ay kinukunsumo ng katawan at hindi na bumabalik sa kani-kanilang pinagmumulan. Pangalawa, para mabigyan niya ng saysay ang nakalilitong presensya ng dalawang ventricle ng puso, ipinaliwanag ni Galen na ang dugong kinargahan na ng buhay mula sa baga ay dumadaloy mula sa kanang ventricle patungo sa kaliwang ventricle sa pamamagitan ng mga maliit na butas ng interventricular septum, $\mathrm{o}$ ang parte ng puso na naghihiwalay sa dalawang ventricle nito.

Naging matagumpay at popular na manggagmot at surgeon si Galen, at dahil napakarami ang kanyang naisulat na akda nagkaroon siya ng maraming tagasunod kahit noong buhay pa siya. Matapos siyang mamatay, namromroblema ang mga tagasunod na ito dahil sa sobrang dami ng kanyang mga akda ay hindi na nila malalaman kung paano ang mga ito ituturo sa mga mag-aaral ng medisina at surgery. Ang niliha nilang solusyon ay humalaw na lamang ng ilang teksto mula sa kanyang mga obra, binansagan itong "Ang Labing-Anim na Libro ni Galen," at ginamit bilang silabus ng edukasyong medikal. ${ }^{16}$ Ang silabus na ito ang dahilan kung bakit Hippokratiko at Galeniko ang kabuoang anyo ng medisina at edukasyong medikal ng Europa hindi lamang sa midyebal na panahon kung hindi pati na rin sa mga unang yugto ng modernong panahon. ${ }^{17}$

\section{Ang Hippokratismo at Galenismo sa Miyebal na Panahon}

\footnotetext{
${ }^{14}$ Armelle Debru, "Physiology," in The Cambridge Companion to Galen, 268.

${ }^{15}$ Joseph Frank Payne, Harvey and Galen (London: Henry Frowde, 1897), 36-48.

${ }^{16}$ Nutton, “The Fortunes of Galen," 362.

${ }^{17}$ Ibid., 371-372.
} 
Dahil ang pagbagsak ng Empiryo Romano noong ika-anim na siglo ay sinundan ng panahon ng kadiliman (dark ages), naging iba ang organisasyon ng medisina sa midyebal na Europa kung ihahambing ito sa minimiti ni Galen na dapat maging iisa ang propesyon ng panggagamot at surgery. Sa miyebal na Europa lalo pang dumami ang nakikihati sa medikal na propesyon: sa isang banda ay ang mga Hippokratiko at Galenikong manggagamot na nagsanay sa mga pamantasang medikal, katulad ng mga pamantasan ng Salerno, Montpellier, Paris at Bologna; sa pangalawang banda ay ang mga surgeon na sa pang-araw-araw na buhay ay nagtratrabaho bilang mga barbero at nakakuha ng kaalaman sa pagsagawa ng mga simpleng operasyon sa pamamagitan ng apprenticeship sa ilalim ng mga nakatatandang barberong surgeon; at sa pangatlong banda ay ang mga herbalista at apotekarista na katulad sa mga barberong surgeon ay hindi rin nakaapak sa pamantasang medikal. Kapag ikinagugulat natin ang kaalamang mga barbero lang pala ang mga surgeon sa midyebal na Europa, dapat sigurong alalahanin natin na ang sagisag ng mga barbero na nakikita pa rin nating nakakabit sa mga pintuan ng kasalukuyang barberya ay nagmula sa imahen ng mga duguan at malinis na bendaheng nakasampay sa labas ng mga midyebal na barberya.

Kapag ang tututukan muna natin ay ang mga kagalang-galang na mga manggagamot na nagmumula sa mga pamantasang medikal, masasabi nating mas Hippokratiko kaysa Galeniko ang anyo ng midyebal na medisina. Ito ay dahil ang mga doktor na ito ay hindi lamang nagkukulang sa kakayahang magsagawa ng surgery, kung hindi minamaliit ng mga ito ang propesyon ng mga barberong surgeon. Dagdag pa dito, ang Griyegong taboo laban sa pagsasagawa ng dissection at vivisection, na noong panahon ni Galen ay sinigundahan na ng Romanong kaugalian, ay lalong pina-igting sa Islamikong paniniwala na ang bangkay ay dapat mailibing kaagad sa loob ng isang araw. Maimpluwensya ang relihiyong Islam dahil nagmula sa mga Arabong manggagamot at intelektuwal ang mga Hippokratiko at Galenikong manuskrito na ginagamit noong midyebal na panahon.

Nang unti-unting namumbalik ang interes ng mga pamantasang medikal sa anatomiya, muling tumingkad ang pagiging Galeniko ng midyebal na medisina. Ngunit ibang klaseng Galenismo ang umiiral sa mga pamantasang ito. Sa halip na isasagawa nila ang dissection para mapatalas ang anatomikal at pisyolohikal na kaalaman ng mga mag-aaral, isinasagawa nila ang kanilang napakadalang na dissection bilang isang ritwal sa pagpapatibay sa otoridad ni Galen sa anatonomiya at pisyolohiya. Kahit sa mga unang yugto ng Renaissance nangyayari pa rin ang tipikal na midyebal na ehersisyo kung saan ang propesor ng anatomiya ay nakaupo sa isang mala-tronong silya at nagbabasa ng akda ni Galen, habang ang kanyang alalay na barbero ay abala sa pagsagawa ng aktuwal na dissection sa harap ng mga mag-aaral na nanonood at nakikinig lamang. ${ }^{18}$ Sa mga pagkakataong hindi

${ }^{18}$ Robinson, The Story of Medicine, 253. 
tumutugma ang resulta o detalye ng isinasagawang dissection sa mga tekstwal na deskripsyon ni Galen, mabilis na ipapaliwanag ng propesor na hindi si Galen ang may problema, at baka may kaunting abnormalidad lamang ang bangkay na kanilang hinihimay. Tila nakalimutan na ng lahat na ang mga hindi pagtutugmang ito ay baka dulot sa katotohanang katawan ng mga hayop na man talaga ang pinagaralan ni Galen.

Mahalagang tandaan natin na ang Hippokratismo at Galenismong umiiral sa midyebal na panahon ay mga diskursong nakabatay sa iilan lamang mga teksto mula sa kabuoang Hippocratic Corpus at sa kabuong mga akda ni Galen. Kadalasan ang mga tekstong ito ay mga salin o komentaryo lamang mula sa mga nauunang salin o komentaryo. Ikinuwento, halimbawa, ni Victor Robinson, sa kanyang librong The Story of Medicine, ang kaparehong nangyayari sa ilang teksto ni Aristotle na kung tutuusin ay "Latin na salin mula sa isang Hebrew na salin na mula sa Arabong komentaryo sa isang Arabong salin na mula sa isang Syriac na salin ng orihinal na Griyegong teksto."19 Sa ganitong kalagayan mahirap sabihin kung ang mga kaalamang nakuha ng mga midyebal na mga Europyano mula sa limitado at isinalin-salin nang mga teksto ay talaga bang tugma sa sistema ng medisinang ipinundar nina Hippokrates at Galen.

\section{Ang Renaissance at ang Umpisa ng Paglubog ng Medisinang Hippokratiko at Galeniko sa Kanluran}

Isa sa mga pundasyunal na pangyayari sa panahon ng Renaissance ay ang malawakang pagsagawa ng mga humanistikong iskolar at mananaliksik ng pagaaral sa mga orihinal na akda ang mga sinaunang Griyego at Romanong manunulat. Binungkal ng mga iskolar at mananaliksik na ito ang mga aklatan at arkibo ng mga monastery, simbahan at palasyo para maghagilap ng mga lumang manuskrito. Marami sa kanila ang marunong magbasa ng Griyego, isang kasanayang unang nakuha ng mga Europyano mula sa mga nagsilikasang Griyegong intelektuwal galing sa lunsod ng Constantinople na sinalakay ng mga Turko noong 1453. Ilan sa mga iskolar at mananaliksik na ito ay gumawa ng mga panibago, sistematikong at direktang pagsasalin ng mga Griyegong manuskrito patungo sa wikang Latin. Kaya kung sa midyebal na panahon limitado lamang ang mga akda nina Hippokrates at Galen at nagmumula pa sa ilang pagsalin-salin ng wika; sa panahon ng Renaissance, dumami ang kanilang mga teksto na nagmumula sa mga panibago, sistematiko at direktang pagsasalin ng mga humanistang iskolar at mananaliksik. Ang pagbabagong ito na nangyari sa larangan ng reproduksyon ng mga teksto ay nagdulot ng malawakang pagbabago sa kultura at kaalaman ng maraming Europyano.

${ }^{19} \mathrm{Ibid} ., 240$.

(C) 2013 F.P.A. Demeterio

http://www.kritike.org/journal/issue 12/demeterio june2013.pdf ISSN 1908-7330 
Dahil sa pagpapahalaga at pagsasalin sa mga Griyegong manuskrito nina Hippokrates at Galen, naganap sa mga unang yugto ng Renaissance ang paglakas ng Hippokratismo at Galenismo. Subalit sa kalaunan, ang pagpapahalaga at pagsasaling ito, na sinabayan ng mapanuri at masinsinang pag-aaral, ay humantong sa mga pagpuna at pagbatikos sa nasabi nang mga teksto. Kaya naganap din sa mga huling yugto ng Renaissance ang paghina ng Hippokratismo at Galenismo matapos maranasan ng mga ito ang ilang malubhang puna at batikos mula kina Paracelsus, Vesalius, Fracastoro at Harvey.

Ang unang pangunahing batikos sa Hippokratiko at Galenikong medisina noong panahon ng Renaissance ay nagmula sa Swisong manggagamot, surgeon at alkemistang si Paracelsus (1493-1541). Hindi malinaw kung gaano kahumanista si Paracelsus dahil madalas siyang nagyayabaang na hindi siya mahilig magbasa ng libro, at na ang kanyang mga kaalman ay nakalap niya mula sa kanyang sariling karanasan at pag-iisip. Ngunit ang pangalang "Paracelsus" na ibinansag niya sa kanyang sarili, na nangangahulugang "Mas mahusay pa kay Celsus," ay tila naaayon sa humanistang pagpapahalaga sa sinaunang Romanong manggagamot at manunulat na si Aulus Cornelius Celsus (circa 25 BC-circa 50 AD).

Philip Theophrastus Bombastus von Hohenheim ang tunay na pangalan ni Paracelsus at ipinanganak siya sa Sihlbrucke, sa Canton ng Schwyz, sa bansang Switzerland. Nakuha niya ang kaalaman tungkol sa medisina, surgery at alkemiya mula sa kanyang ama. Pinalalim niya ang kanyang alkemiya sa patnubay ng isang Benediktinong Abad na si Joannes Trithemius (1415-1516), at nag-aral ng metalurhiya sa ilalim ng isang may pangalang Sigmund Fugger. Higit sa lahat pinayaman niya ang kanyang pananaw at kaisipan sa pamamagitan ng paglalakbay at pakikipagsalamuha hindi lamang sa ibang manggagamot, surgeon at alkemista, kung hindi pati na rin sa ilang mga sundalo, berdugo, barbero, pastol, gypsy, komadrona, at manghuhula. ${ }^{20}$ Ang kanyang kawalan ng tiwala sa klasikong medisina ay simboliko niyang inihayag sa pamamagitan ng pagsunog sa mga libro nina Galen at Avincenna doon sa lunsod ng Basel.

Ang batikos ni Paracelsus sa Hippokratiko at Galenikong medisina ay nakasentro sa kanilang teorya ng kalusugan, sakit at karamdaman, at panggagamot, na lahat ay nakabatay sa doktrina ng apat na humour. Sa kanyang paliwanag tungkol sa sanhi ng mga sakit at karamdaman, isinantabi ni Paracelsus ang doktrinang ito, at naglahad ng kanyang sariling teorya tungkol sa limang sanhi: ang ens astrale, $\mathrm{o}$ ang kosmiko at klimatikong mga impluwensya sa katawan ng tao; and ens venenale, or mga nakalalasong materyal na nakukuha ng tao mula sa pagkain at sa kapaligiran; ang ens naturale, or ang mga nakahahawang sakit, na noon ay

${ }^{20}$ Franz Hartmann, The Life of Philippus Theophrastus Bombast of Hohenheim Known by the Name of Paracelsus and the Substance of his Teachings Concerning Cosmology, Anthropology, Pneumatology, Magic, Sorcery, Medicine, Alchemy and Astrology, and Philosophy and Theosophy (London: Kegan Paul, Trench, Trubner and Company Limited, 1896), 5.

(C) 2013 F.P.A. Demeterio

http://www.kritike.org/journal/issue 12/demeterio june2013.pdf

ISSN 1908-7330 
isinisisi nila sa miasma, o mga binhhi ng sakit at karamdaman na lumulutang sa hangin; ang ens spirituale, o ang hindi magandang mental, o sikolohikal, na disposisyon; at ang ens deale, o mga karamdamang ipinadala mula sa kalangitan. ${ }^{21}$ Itinuturo ni Paracelsus na ang isang sakit ay maaaring isang epekto ng isa, dalawa o higit pa sa mga sanhing ito. Kaya dapat lamang na ang isang manggagamot ay may alam sa astrolohiya para kaya niyang tugunan ang unang sanhi (ens astrale), sa alkemiya para kaya niyang labanan ang ikalawa (ens venenale) at ikatlong sanhi (ens naturale), sa teorya tungkol personalidad at pagkatao para kaya niyang hanapan ng solusyon ang ika-apat na sanhi (ens spirituale), at sa teolohiya para kaya niyang harapin ang ikalimang sanhi (ens deale).

Bilang lunas sa mga sakit at karamdamang dulot ng ens venenale at ens naturale, iginigiit ni Paracelsus na sa halip na umasa sa mga malamyang herbal na gamot, maghahanap dapat ang alkemiya ng mga naaangkop na kemikal na gamot. Isa sa mga pinaka kilalang preskripsyon ni Paracelsus ay ang asoge (mercury) bilang lunas sa sakit na syphilis. Alam niya ang panganib na maaaring idudulot ng mga kemikal na droga, kaya binabantaan niya ang kanyang mga tagasunod na magiingat sa pagpasya kung gaano kadami ang ipapainum nila sa kani-kanilang mga pasyente. Kapag hindi sapat ang dami ng droga ay wala itong itong silbi, ngunit kapag sobra naman ang dami nito ay magiging lason naman ito na maaaring papatay sa mga may sakit at karamdaman.

Dalawa ang naging pangunahing batikos ni Paracelsus sa nagpapahina sa medisina nina Hippokrates at Galen. Una dito ang kanyang paliwanag na ang mga sakit at karamdaman ay maaaring mga epekto ng mga eksternal na sanhi. Ito ay isang direktang atake sa noon ay napakatatag na doktrina ng apat na humour. Pangalawa dito ay ang kanyang paghatak sa alkemiya patungo sa larangan ng medisina para makapagbigay ng mga bago at matatapang na lunas na nasa labas ng diskurso ng pagpapanumbalik sa natural na balanse ng mga humour.

Nakalulungkot isipin na kahit pa man radikal at makapangyarihan ang mga suhestyon ni Paracelsus para sa modernisasyon ng medisina, hindi siya nagkaroon ng malawakang impluwensya sa labas ng mga Alemanikong estado. Ito ay bunsod na siguro sa kanyang personalidad na mapusok, matapang at may kayabangan na nagdulot sa kanya ng mas maraming kalaban at kagalit kaysa mga kaalyado. Ito ay pinalala pa sa kanyang walang tigil na paglalakbay sa Europa, Afrika at Asia Mino na humadlang sa anumang posibilidad ng pagkakaroon ng isang matatag na pangkat ng mga tagasunod. Gayunpaman ang mga batikos ni Paracelsus ay tumatayong pambungad na pasabog sa mga susunod pang mga atake laban sa Hippokratismo at Galenismo.

Ang ikalawang pangunahing batikos sa Hippokratiko at Galenikong medisina noong panahon ng Renaissance ay nagmula sa Flemish na manggagamot

${ }^{21}$ Ibid., 199-228.

(C) 2013 F.P.A. Demeterio

http://www.kritike.org/journal/issue 12/demeterio june2013.pdf ISSN 1908-7330 
at surgeon na si Andries van Wesel (1514-1564), o mas kilala bilang Andreas Vesalius. Hindi katulad kay Paracelsus, isang tunay na humanistang iskolar si Vesalius na nakapagsagawa ng ilang obra tungkol kay Galen at iba pang sinaunang manunulat. ${ }^{22}$ Ipinanganak si Vesalius sa Brussels, sa bansang Belgium, kung saan nanungkulan ang kanyang ama bilang apotekarista sa Imperyal na korte ni Carlos V. Nag-aral siya sa lunsod ng Louvain at lumipat sa lunsod ng Paris, kung saan naging guro niya ang mga batikang Galenista na sina Johannes Quinterus ng Andernach (1505-1574) at Jacobus Sylvius (1478-1555) na pinamangha niya sa kanyang kasanayan sa anatomiya. Nakamtan niya ang kanyang titulo sa medisina mula sa Pamantasan ng Padua.

Ang batikos ni Vesalius sa Hippokratiko at Galenikong medisina ay nakasentro sa anatomiya ni Galen. Nag-umpisa si Vesalius bilang isang masugid na tagasunod sa Galenistang anatomiya at nagsagawa siya ng masinsinang pagaaral sa mga teksto ni Galen hindi lamang sa pamamagitan ng mga tekstual na istratehiya, kung hindi pati na rin sa pamamagitan ng mabusising paghahambing sa mga anatomikal na deskripsyon ni Galen at sa mga datos na kanyang nakalap mula sa kanyang mga sariling dissection. ${ }^{23}$ Sinasabi ng ilang historyador na dahil sa dedikasyon ni Vesalius sa pagsasagawa ng dissection humantong siya sa puntong siya mismo ang namumulot ng mga bangkay mula sa bitayan ng Montfaucon at sa Sementeryo ng mga Inosente. ${ }^{24}$

Labis ang pagkagulat ni Vesalius nang madiskubre niya na ang mga anatomikal na akda ni Galen ay nakabatay pala sa dissection at vivisection ng mga hayop, isang proseso na noon ay hindi naman itinatago ni Galen ngunit tuluyang nabaon sa limot pag sapit ng midyebal na panahon. ${ }^{25}$ Dahil sa kanyang natuklasang "sekreto" ni Galen, naisip ni Vesalius na sa halip na pagkakatiwalaan ang dakilang Griyego-Romanong pantas ng anatomiya, dapat pala siyang pagdududahan. Kapag tuluyan nang kumupas ang otoridad ni Galen, binigyang diin ni Vesalius na wala nang iba pang maaasahan ang mga propesor at mag-aaral sa anatomiya kung hindi ang kani-kanilang aktuwal at masinsinang pagsasagawa ng dissection at vivisection.

Natukoy ni Vesalius ang higit dalawang-daang pagkakamali ni Galen, at itinuwid niya ang mga ito sa kanyang mala-bantayog na obrang On the Workings of the Human Body. Ang obra maestrang ito ay naglalaman ng maraming realistiko at kaaya-ayang ilustrasyon na iginuhit ni Johann Stephan von Calcar (1499-1546), isang pintor na nagsanay sa talyer ni Titian (1488-1576). Ito ang kauna-unahang aklat sa anatomiya kung saan ang katawan ng tao ay sinuri sa isang lohikal na

\footnotetext{
${ }^{22}$ Robinson, The Story of Medicine, 253.

${ }^{23}$ John Farquhar Fulton, Logan Clendening Lectures on the History and Philosophy of Medicine (Lawrence, Kansas: University of Kansas Press, 1950), 9.

${ }^{24} \mathrm{Ibid}$.

${ }^{25}$ Nutton, "The Fortunes of Galen," 386.
} 
paraan mula sa kalansay palabas. Pati ang masining na pagrerepresenta sa realistikong anyo ng anatomiya ng katawan ay nagtakda ng bagong pamantayan sa anatomikal na ilustrasyon na dati ay nakukuntento na sa mga malalabong diyagramatikong representasyon. Dahil sa inobasyong ito naging mas espesipiko at malinaw ang mga diskusyon tungkol sa anatomiya.

Isa sa mahigit dalawang-daang pagkakamali ni Galen na tinukoy ni Vesalius ay ang tungkol sa mga butas ng interventricular septum, ang parte ng puso na naghihiwalay sa dalawa nitong ventricle. Matatandaan nating ipinilit ni Galen na may butas ang septum na ito para maipaliwanag niya ang kanyang inakalang paglipat sa dugong kargado na ng buhay mula sa baga mula sa kanang ventricle patungo sa kaliwang ventricle. Pinatunayan ni Vesalius na wala siyang nakitang mga butas sa septum na ito, at na imposibleng magkakaroon ng butas sa parteng ito ng puso. Ang implikasyon ng tila maliit na detalyeng ito sa puso ng tao ay ang pagsantabi sa teorya ng daloy ng dugo na inilahad na Galen.

Hindi nagkaroon ng pagkakataong makapaglahad ng panibagong teorya ng daloy ng dugo si Vesalius dahil na siguro sa naging maikli lamang ang kanyang panunungkulan bilang propesor sa anatomiya at medisina bunsod na rin sa mga matitinding batikos na naranasan niya mula sa mga masugid na Galenista. Gayunpaman, ang kanyang pagpasinungaling sa konsepto ng butas-butas na interventricular septum ay lumikha ng pangangailangan para sa isang bagong teorya tungkol sa daloy ng dugo.

Ang pangunahing batikos ni Vesalius na nagpapahina sa medisina nina Hippokrates ay ang kanyang pagpuna sa otoridad ni Galen sa larangan ng anatomiya kasabay ang suhestiyong dapat magkaroon ng tiwala ang mga anatomista sa kani-kanilang sariling pagkalap ng panibagong anatomikal na datos. Taliwas sa inaakala ng ibang historyador, ang pagpasinungaling ni Vesalius sa Galenikong teorya tungkol sa daloy ng dugo ay hindi nagkaroon ng malalim na epekto sa Hippokratiko at Galenikong doktrina ng apat na humour. Malaki ang ibinuwis ni Vesalius kapalit sa kanyang pagbatikos sa Galenikong anatomiya. Dahil sa galit ng mga Galenista sa kanya iniwan niya ang akademya at nanilbihan na lamang bilang manggagamot sa imperyal na korte ni Carlos V. Isang araw, habang nagsasagawa siya ng isang dissection sa harap ng ilang manonood, nagulat ang lahat matapos nilang makita ang tumitibok pa na puso ng inakala nilang bangkay. Sinampahan siya ng kasong pamamaslang ng mga Galenista. Subalit dahil malapit siya sa imperyal na korte ang kanyang kaparusahan ay pinagaan sa anyo ng pagsagawa ng peregrinasyon sa Herusalem. Sa kakapusang palad, namatay siya sa isang sakit habang pauwi na sana sa Europa.

Ang ikatlong pangunahing batikos sa Hippokratiko at Galenikong medisina noong panahon ng Renaissance ay nagmula sa Italyanong manggagamot, dalubhasa sa matematika, heograpiya at astronomiya, at makatang si Girolamo Fracastoro (1478-1553), o Hieronymus Fracastorius. Katulad kay Vesalius, tunay 
din na humanista si Fracastoro, at ito ay kanyang ipinapamalas hindi sa pamamagitan ng pagasalin at pag-edit ng mga sinaunang manuskrito, kung hindi sa pamamagitan ng kanyang malawak na interes sa iba't ibang larangan, o pagiging "uomo universale" sa wikang Italyano. Ipinanganak si Fracastoro sa lunsod ng Verona, na noon ay sakop ng Republica ng Venecia, at una siyang nag-aral ng literatura at pilosopiya sa ilalim ng kanyang ama, at nag-pakadalubhasa sa literatura, pilosopiya, matematika, astronomiya at medisina sa Pamantasan ng Padua. Dahil sa kanyang kahusayan sa panggagamot, pinili siyang pangunahing manggagamot ng mga unang session ng Konseho ng Trent (1545-1547).

Hindi man direktang binatikos ni Fracastoro ang Hippokratiko at Galenikong medisina, subalit ang kanyang pagsuri sa sanhi ng mga nakahahawang sakit, katulad ng syphilis na siya pa ang nagbigay ng pangalan, ng typhus, at ng salot, ay nagtatag ng isang malakas na alternatibong teorya sa sakit at karamdamang dati ay nakabatay lamang sa doktrina ng apat na humour. Mas espesipiko at detalyo ang diskurso ni Fracastoro tungkol sa mga sanhi ng nakahahawang sakit kaysa diskusyon ni Paracelsus tungkol sa kanyang konsepto ng ens naturale na binase lamang sa lumang ideya ng miasma, o masamang hangin.

Itinuturo ni Fracastoro na paghawa ng sakit ay maaaring maganap sa pamamagitan ng tatlong paraan: direktang kontak, kung kailan hinawakan o dinikitan ng taong walang sakit ang taong may sakit; hindi direktang kontak, kung kailan hinawakan ng taong walang sakit ang mga damit o anumang kagamitan ng taong may sakit; at ang paghawa kahit walang anumang kontak, kung kailan nalanghap ng taong walang sakit ang hangin na nakapalibot sa tao o mga taong may sakit. ${ }^{26}$ Ang pinaka-ugat sa proseso ng paghawa ng sakit ay ang tinatawag ni Fracastoro na "seminaria contagionis," o "butil ng pagkakahawa." Noong hindi pa naimbento ang mikroskopyo, naisip na ni Fracastoro na ang sanhi ng mga nakahahawang sakit ay ang mga hindi nakikitang butil na nariyan sa katawan ng taong may sakit, o lumulutang-lutang sa hangin na kapag dumapo na sa sistema ng taong walang sakit ay bigla na lamang dumadami at lilikha ng mga sintomas ng partikular na sakit at karamdaman. Espesipiko ang mga seminaria contagionis, sa puntong ang mga hindi nakikitang butil ng syphilis ay hindi maaaring magdudulot ng typhus, o ang mga hindi nakikitang butil ng ketong ay hindi maaaring magdudulot ng malaria.

Ang teorya ni Fracastoro tungkol sa sanhi ng sakit at karamdaman ay may kasamang teorya sa panggagamot. Dahil natukoy na niya na ang mga sakit at karamdaman ay maaaring epekto ng seminaria contagionis, nararapat lamang na maghahanap ang mga manggagamot ng paraan para puksain ang mga butil na ito, o kung hindi man para pigilan ang pagdami ng mga ito sa loob ng katawan. Dahil

${ }^{26}$ Vivian Nutton, “The Reception of Fracastoro's Theory of Contagion: The Seed That Fell among Thorns?," in Osiris, 6:2 (1990), 200.

(C) 2013 F.P.A. Demeterio

http://www.kritike.org/journal/issue 12/demeterio june2013.pdf

ISSN 1908-7330

\section{(cc) BY-NC}


espesipiko ang mga butil na ito, dapat espesipiko din ang paraan sa pagpuksa sa kanila, o sa pagpigil sa kanilang pagdami. ${ }^{27}$

Kahit pa man hindi niya direktang kinalaban sina Hippokrates at Galen, ipinakita ni Fracastoro na may malaking pagkukulang ang mga ito sa puntong hindi nila hawak ang lahat ng sagot tungkol sa kung paano labanan ang mga nakahahawang sakit. Dahil sa pagkadiskubre ni Fracastoro na ang mga nakahahawang sakit ay epekto ng mga espesipikong seminaria contagionis, dapat tigilan na muna ng mga manggagamot ang kanilang pagpapakadalubhasa sa doktrina ng apat na humour, at magpursige silang pag-aralan ang mga espesipikong nakahahawang sakit para mahanapan nila ang mga ito ng mga espesipikong lunas.

Ang ika-apat na pangunahing batikos sa Hippokratiko at Galenikong medisina noong panahon ng Renaissance ay nagmula sa Ingles na manggagamot at anatomistang si William Harvey (1578-1657), o Gulielmus Harveius. Ipinanganak si Harvey sa county ng Kent sa isang mayaman at edukadong angkan, nagtapos sa kanyang araling batsilyer sa Pamantasan ng Cambridge, at katulad kina Vesalius at Fracastoro ay nakakuha ng kanyang titulo sa pisyolohiya mula sa tanyag na Pamantasan ng Padua. Naging guro niya sa Pamantasang ito ang batikang Italyanong anatomista, surgeon, at ama ng embriyolohiya na si Girolamo Fabrizio (1537-1619), na mas kilala bilang Fabricus ab Aquapendende, ${ }^{28}$ na minsan naman ay naging mag-aaral ni Vesalius. Bumalik si Harvey sa Inglaterra para makamtan ang kanyang titulo sa medisina mula sa Pamantasan ng Cambridge at para manungkulan bilang manggagamot at propesor ng medisina. Naging personal siyang manggamot ng mga Ingles na Haring Jaime I at Carlos I.

Ang batikos ni Harvey sa Hippokratiko at Galenikong medisina ay nakasentro sa teorya ng daloy ng dugo ni Galen na una nang pinasinungalingan ni Vesalius sa pamamagitan ng kanyang demonstrasyon na walang mga butas ang interventricular septum. Sa mas madaling salita, si Harvey ang lubusang nakinabang sa puwang na iniwan ni Galen matapos siyang isinantabi ni Vesalius.

Tuluyang ibinasura ni Harvey ang Galeniko at Aristotelyang doktrina tungkol sa mga dugong nagmumula sa puso, o dugong nagdadala ng buhay, at sa atay, o dugong nagdadala ng sustansya, na parehong kinukunsumo ng iba't ibang parte ng katawan. Gamit ang kaalaman mula kay Vesalius na hindi maaaring lumipat ang dugo mula sa kanan patungo sa kaliwang ventricle dahil walang mga butas ang interventricular septum na puwede nitong dadaanan, at gamit ang kaalaman mula kay Fabricus tungkol sa mga barbula ng artery at vein na nagpapadaloy sa dugo sa isang direksyon lamang, inilahad ni Harvey ang kanyang teorya tungkol sa sirkulasyon ng dugo. ${ }^{29}$

\footnotetext{
${ }^{27}$ Ibid., 203.

${ }^{28}$ D'Arcy Power, William Harvey (London: T. Fisher Unwin, 1897), 15.

${ }^{29}$ Ibid., 25.
} 
Ang teorya ng sirkulasyon ng dugo ni Harvey ay binubuo ng dalawang rota: ang pulmonar na sirkulasyon at ang mas malawak na sistemikong sirkulasyon. Ang pulmonar na sirkulasyon ay nagaganap habang ang dugong mababa na ang dalang oxygen ay napupunta sa kanang ventricle ng puso at binubomba patungo sa baga para kakargahan ng oxygen, at ito ay babalik sa kaliwang ventricle ng puso. Ang sistemikong sirkulasyon naman ay nagaganap habang ang dugong kargado na ang oxygen ay binubomba palabas sa kaliwang ventricle ng puso patungo sa iba't ibang parte ng katawan gamit ang mga artery, at ito ay babalik sa kanang ventricle ng puso gamit ang mga vein. Inihayag ni Harvey ang konsepto ng dalawang sirkulasyon kahit pa man hindi niya lubusang naintindihan kung ano ang nagdudugtong sa mga artery at vein. Lilipas muna ang mahigit kumulang kalahating siglo bago matukoy ng Italyanong manggagamot at anatomistang si Marcello Malpighi (1628-1694) ang mga capillary na aktuwal na nagdudugtong sa mga artery at vein gamit ang kanyang mikroskopyo. ${ }^{30}$

Katulad sa ginawa ni Vesalius, ang pangunahing batikos ni Harvey na nagpapahina sa medisina nina Hippokrates ay ang kanyang pagpuna sa otoridad ni Galen sa larangan ng anatomiya kasabay ang suhestiyong dapat magkaroon ng tiwala ang mga anatomista sa kani-kanilang sariling pagkalap ng panibagong anatomikal na datos. Sa katunayan minsan nang isinulat ni Harvey ang pahayag na "Isinusumpa ko na mag-aral at magturo ng anatomiya, hindi batay sa mga libro kung hindi batay sa mga dissection, hindi batay sa kung ano ang sinasabi ng mga pilosopo kung hindi batay sa sinasabi ng tela ng kalikasan (fabric of nature). ${ }^{\text {"31 }}$ Taliwas sa inaakala ng ibang historyador, ang pagpalit ni Harvey sa Galeniko at Aristotelyang teorya ng daloy ng dugo ng kanyang sariling teorya sa sirkulasyon ng dugo ay hindi pa rin nagresulta sa agarang pagsantabi sa Hippokratiko at Galenikong doktrina ng apat na humour. Isinulat ni Vivian Nutton, sa kanyang sanaysay na "The Fortunes of Galen," na "sa katunayan, ang Galenikong teorya sa panggagamot, lalo na ang venesection at paggamit ng droga, ay maipapaliwanag ng mas maayos gamit ang pisyolohiya ni Harvey kaysa gamit ang pisyolohiya ni Galen. Maski si Harvey ay walang nakitang dahilan na baguhin ang kanyang nakagisnan nang metodo sa panggagamot." ${ }^{32}$

Hindi lubusang naganap ang siyantipikong rebolusyon sa larangan ng medisina sa loob ng panahon ng Renaissance, ang tanging nagawa nina Paracelsus, Vesalius, Fracastoro at Harvey ay ang pagpapahina lamang sa dogmatikong otoridad nina Hippokrates at Galen, na nagbukas naman ng maraming pag-aaral at pananaliksik na hindi na nakabatay sa klasikong mga teksto kung hindi sa mga aktuwal at mapanuring dissection, vivisection at eksperimentasyon. Ayon kay Nutton ang tuluyang nagsantabi sa Hippokratiko at Galenikong teorya ng

\footnotetext{
${ }^{30}$ Ibid., 190.

${ }^{31}$ Ibid., 90.

${ }^{32}$ Nutton, “The Fortunes of Galen," 376.
} 
panggagamot ay ang pag-yabong ng kemikal na parmakolohiya, na unang ipinundar ni Paracelsus, ang mekanistikong pagdadalumat ng katawan, sakit at karamdaman ng mga iatromechanist, ang pagdagsa ng mga bagong herbal na gamot mula sa Amerika at Indies, ang mga pagbabago sa klinikal na panggagamot at pisyolohiya, at higit sa lahat ang mas mabilis na takbo ng buhay na dulot ng industriyalisasyon na sumasalungat sa mabagal, mabusisi at napakapersonal na paraan ng panggagamot nina Hippokrates at Galen. ${ }^{33}$

\section{Kongklusyon}

Katulad ng nabanggit na sa introduksyon ng sanaysay na ito, ang huling seksyon na ito ay tutugon sa mga katanungang ano ang mga aspekto na nagsisilbing dahilan kung bakit tunay ngang matatawag na rebolusyunaryo ang mga nangyayaring pagbabago sa larangan ng medisina noong panahon ng Renaissance, kung ano ang mga elemento ng tradisyunal na medisina ang naisantabi at nanatili, at kung paano naiiba ang proseso ng pagpalit ng paradigma sa larangan ng medisina sa kapareho ngunit mas kilalang proseso sa larangan ng astromiya at pisika?

Kahit pa man hindi kasing dramatiko at naging ganap ang mga pagbabago sa larangan ng medisina kapag ihahambing sa mga pagbabago sa larangan ng astronomiya at pisika noong panahon ng Renaissance, masasabi pa rin nating tunay na rebolusyunaro ang mga ito, dahil sa pamamagitan ng mga historikal na naratibong nabuo natin, naipakita natin kung paano lumubog, na parang araw, ang hegemony nina Hippokrates at Galen sa mundo ng produksyon ng kaalamang medikal at pisyolohikal. Ang dapit hapong sumapit sa paglubog nina Hippokrates at Galen ay sinabayan naman ng madaling araw kung kailan sumikat ang liwanag na dala ng maraming siyantista at ng kanilang sariwang pananaliksik na sa kalaunan ay lumikha ng panibagong diskurso sa larangan ng medisina.

Naibasura man ng siyantipikong rebolusyon ang mga teorya ng kalusugan, ng sakit at karamdaman, at ng panggagamot nina Hippokrates at Galen, pati na ang anatomiya ni Galen, subalit may dalawang mahalagang elemento mula sa dalawang pantas na ito ang nanatili sa modernong mundo ng medisina. Una dito ay ang pilosopiya at etika ni Hippokrates na nagtuturo kung paano dapat kikilos at mag-iisip ang isang mahusay at mabuting manggagamot. Ang aspektong ito ay simbolikong inuukit sa kamalayan ng mga bagong manggagamot sa panahon ng kanilang panunumpa. Pangalawa dito ay ang pagpapahalaga ni Galen sa anatomiya at pisyolohiya para sa pagpapaunlad ng medisina. Noong nakatali pa siya sa teorya ng panggagamot ni Hippokrates na nakabatay sa doktrina ng apat na humour, hindi lubusang maipaliwanag ni Galen kung bakit napakahalaga ang anatomiya at pisyolohiya sa panggagamot. Ngunit matapos maisantabi ng

$$
{ }^{33} \mathrm{Ibid} ., 379 .
$$


siyantipikong rebolusyon ang doktrina ng apat na humour, pati na ang kanyang sariling anatomiya at pisyolihiya, naging malinaw na malinaw na para sa lahat na ang medisina ay dapat ngang nakabatas sa isang masinsinang pag-aaral sa anatomiya at pisyolohiya.

Naiiba ang anyo ng siyantipikong rebolusyon sa larangan ng medisina kapag ihahambing ito sa siyantipikong rebolusyon sa larangan ng astronomiya. Una, kapag titingnan natin ang timeline na naghahayag sa pagkakalimbag ng mga pangunahing libro na bumuo sa siyantipikong rebolusyon sa dalawang larangang ito, dramatiko at agarang naging ganap ang helyosentrismong unang inilahad ng Polako-Alemang astronomong si Nicolaus Copernicus (1473-1543), na sinigundahan ng Italyanong pisisista at astronomong si Galileo Galilei (15641642) gamit ang mga datos na kanyang nakalap sa pamamagitan ng kanyang mga teleskopyo, na binigyan ng mas matalas na modipikasyon ng Alemang matematiko at astronomong si Johannes Kepler (1571-1630), at binigyan ng lehitemasyon ng Ingles na pisisista, matematiko, astronomo at alkemistang si Isaac Newton (16421727).

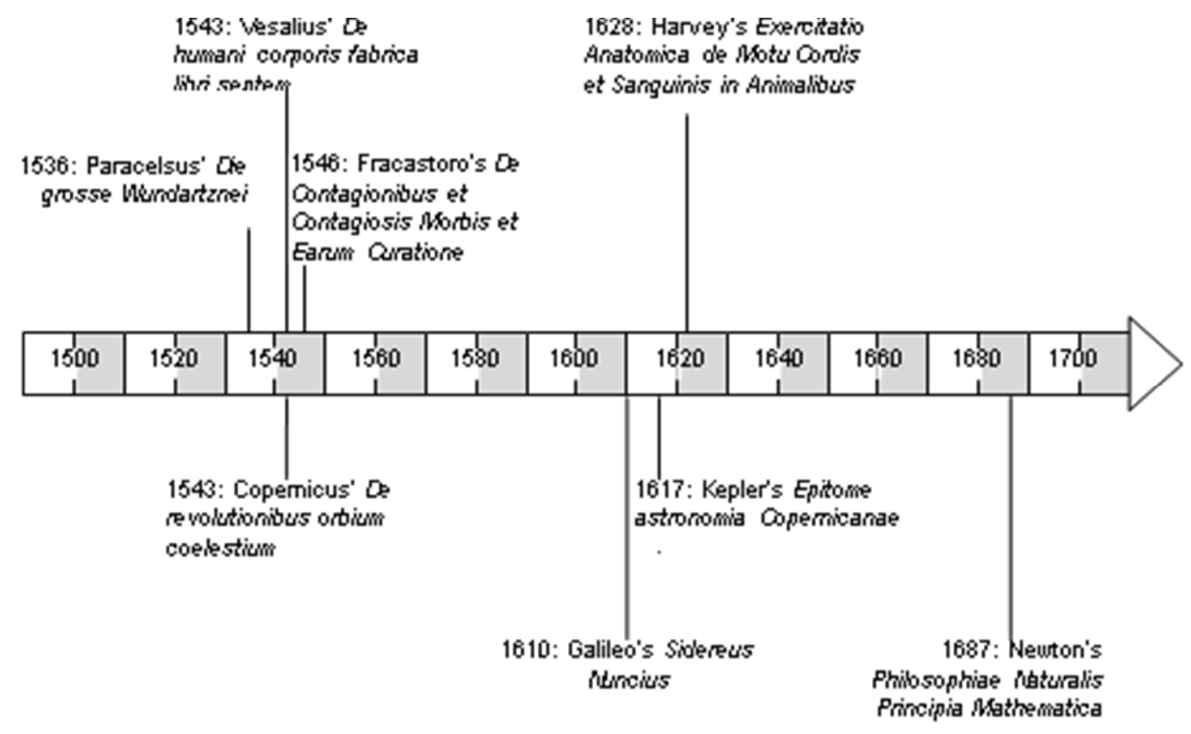

Hugis 1: Timeline ng Pagkakalimbag ng mga Pangunahing Libro na Bumuo sa Siyantipikong Rebolusyon sa mga Larangan ng Medisina at Astronomiya

Samantalang buong teorya kaagad ang inilahad ni Copernicus laban sa lumang sistema ng Romanong Egyptian na si Cladius Ptolemy (circa 90 AD-circa $168 \mathrm{AD}$ ), pira-pirasong mga teorya lamang ang ibinato nina Paracelsus, Vesalius,

(C) 2013 F.P.A. Demeterio

http://www.kritike.org/journal/issue 12/demeterio june2013.pdf

ISSN 1908-7330 
Fracastoro at Harvey laban kina Hippokrates at Galen. Samantalang nagkaroon ng buong kaganapan ang siyantipikong rebolusyon sa astronomiya sa paglathala ni Newton ng kanyang Philosophiae Naturalis Principia Mathematica, hindi ito nangyari sa larangan ng medisina dahil umabot pa ang doktrina ng apat na humour nina Hippokrates at Galen sa panahon ng industriyal na rebolusyon.

Pangalawa, malinaw at malawak ang sentral na paradigma ng Ptolemikong astronomiya na binaklas at pinalitan ni Copernicus. Sa larangan ng medisina, dalawa ang pangunahing paradigma nina Hippokrates at Galen na namamayagpag noon: ang paradigma tungkol panggagamot na nakabatay sa doktrina ng apat na humour at ang paradigma sa tungkol sa anatomikal at pisyolohikal na istraktura ng katawan ng tao. Samantalang dinanas ng ikalawang paradigma ang krisis at rebolusyon, sa wika ni Kuhn, noong mga panahon nina Vesalius at Harvey, hindi ito malinaw na naransan ng unang paradigma. Kaya kahit noong ibinasura na ang anatomiya at pisyolohiya ni Galen, ay nagpatuloy pa rin sa pag-iral ang teorya ng kalusugan at panggamot nina Hippokrates at Galen.

Department of Filipino, De La Salle University-Manila, Philippines

\section{References}

Almario, Virgilio, Patnubay sa Pagsasalin (Manila: Pambansang Komisyon sa Kultura at mga Sining, 1996).

Debru, Armelle, "Physiology," in The Cambridge Companion to Galen (Cambridge: Cambridge University Press, 2008).

Eichholz, D.E., "Galen and his Environment," in Greece and Rome 20:59 (June 1951).

Farquhar Fulton, John, Logan Clendening Lectures on the History and Philosophy of Medicine (Lawrence, Kansas: University of Kansas Press, 1950).

Frank Payne, Joseph, Harvey and Galen (London: Henry Frowde, 1897).

Hartmann, Franz, The Life of Philippus Theophrastus Bombast of Hohenheim Known by the Name of Paracelsus and the Substance of his Teachings Concerning Cosmology, Anthropology, Pneumatology, Magic, Sorcery, Medicine, Alchemy and Astrology, and Philosophy and Theosophy (London: Kegan Paul, Trench, Trubner and Company Limited, 1896).

Henry, John, The Scientific Revolution and the Origins of Modern Science (New York: Palgrave, 2002).

Jones, W.H.S., "General Introduction," in The Loeb's Classical Library's Hippocrates, Vol. 1 (London: William Heinemann Limited).

Nutton, Vivian, "The Fortunes of Galen," in Hankinson, R.J. The Cambridge Companion to Galen (Cambridge: Cambridge University Press, 2008). 
, "The Reception of Fracastoro's Theory of Contagion: The Seed That Fell among Thorns?," in Osiris, 6:2 (1990).

Power, D’Arcy, William Harvey (London: T. Fisher Unwin, 1897).

Robinson, Victor, The Story of Medicine (New York: The Home Library, 1943).

Rocca, Julius, “Anatomy," in The Cambridge Companion to Galen (Cambridge: Cambridge University Press, 2008).

Spina, Francesca, "The Medicine of Ancient Greece," in Facolta di Medicina e Chirurgia dell' Università degli Studi di Cagliari, <http://pacs.unica.it/biblio/lesson1.htm>, Date Accessed: 27 July 2011.

Van der Eijk, Philip, "Therapeutics," in The Cambridge Companion to Galen (Cambridge: Cambridge University Press, 2008). 